\title{
The Use of Ultrasound Images in Manual Therapy and Additionally in Assessment of Shoulder Impingement Syndrome
}

\author{
Luca Collebrusco ${ }^{1,2}$, Rita Lombardini' ${ }^{3}$, Giovanna Censi ${ }^{4}$ \\ ${ }^{1}$ Research Department C.R.O.M.O.N.-EDUCAM School, Rome, Italy \\ ${ }^{2}$ Rehabilitation Unit-National Health Service of Umbria, Perugia, Italy \\ ${ }^{3}$ Dept. of Medicine-University of Perugia, Perugia, Italy \\ ${ }^{4}$ INRCA University Polytechnic of Marche, Ancona, Italy \\ Email: luca.collebrusco@unipg.it
}

How to cite this paper: Collebrusco, L., Lombardini, R. and Censi, G. (2017) The Use of Ultrasound Images in Manual Therapy and Additionally in Assessment of Shoulder Impingement Syndrome. Open Journal of Therapy and Rehabilitation, $\mathbf{5}$, 53-70.

https://doi.org/10.4236/ojtr.2017.52006

Received: March 3, 2017

Accepted: May 16, 2017

Published: May 19, 2017

Copyright $\odot 2017$ by authors and Scientific Research Publishing Inc. This work is licensed under the Creative Commons Attribution International License (CC BY 4.0).

http://creativecommons.org/licenses/by/4.0/

\begin{abstract}
The use of the ultrasound imaging (USI) in physiotherapy is becoming increasingly common but is highly operator dependent and there are safe and professional issues regarding its practical use. Currently there are no specific training guidelines relating to physiotherapists using USI. The use of ultrasound technology for medical applications began in the 1950s and has proven to be an effective, safe, non-invasive, and relatively inexpensive tool for assessing morphologic characteristics and structural integrity of visceral organs and soft tissues. The use of ultrasound to assess muscle morphology and guide rehabilitation decision-making in physical therapy practice can be traced back to the late 1960s and has been found to be reliable and valid for specific muscles during particular movements. Over the last decade there has been rapid development of this technique with increased use both by clinicians and researchers. This method is defined in literature with the denomination of Rehabilitative Ultrasound Imaging (RUSI). In this work we will see how RUSI could be of help in the evaluation of shoulder impingement syndrome (SIS).
\end{abstract}

\section{Keywords}

Rehabilitation, Sonography, Physical Therapy, Ultrasound Imaging, Manual Therapy, Shoulder Pain, Shoulder Impingement Syndrome

\section{Introduction}

Shoulder disorders are second only to low back pain as the most common musculoskeletal disorder, with shoulder impingement syndrome (SIS) being the 
most prevalent [1].

SIS is described as pain or pathology located in the rotator cuff tendons, subacromial bursa, and sub-acromial space [2].

There are many factors connected with the pathogenesis of SIS; repetitive use of the shoulder muscles, incorrect scapulothoracic rhythm, instability of the glenohumeral joint, degeneration of the rotator cuff tendons, and altered shapes of the acromion [3] [4].

Patients with SIS have been found to have decreased strength during resisted external rotation of the shoulder and a significantly elevated position of the humeral head during arm elevation when compared to individuals without shoulder pain.

Functional alterations of the deltoid and rotator cuff during shoulder activities have also been reported in patients with SIS which could lead to a humeral head superior translation.

It is hypothesized that the decreased function of the infraspinatus, as seen in SIS, contributes to the production of SIS pain.

The results of current research suggest that real-time ultrasonography is a reliable method for measuring and studying muscle movement of the cuff rotator and scapula and for assessing the incidence of dyskinesia in specific cases of motor control impairments.

The evaluation of muscles in different conditions ofrest and contractile states may help us to prevent shoulder pain from syndrome impingement [5] [6].

Clinical useof Rehabilitation Ultrasound Imaging (RUSI) is related to interventions aimed at addressing these specific motor control impairments.

\section{Overview RUSI}

Over the past 30 years, increasing numbers of physical therapists have employed conventional grayscale brightness mode (B-mode) ultrasound imaging (USI) to assess components of muscle morphology (the form and structure of muscle) and morphometry (measurements of muscle form or size) as a means to speculate on muscle function in both research and clinical settings [7] [8] [9].

To that end, the term "rehabilitative ultrasound imaging" (RUSI) was coined as a means to encompass and define these applications [10].

Specifically, RUSI refers to USI procedures used by physical therapists to evaluate the morphology and behavior (morphometry) of muscle and related soft tissues, to provide biofeedback about muscle morphometry during restoration of function and to carry out research aimed at informing clinical practice [11].

RUSI has been advocated to improve the understanding of the relationship between motor control and function, determine which patients may benefit from a specific exercise treatment approach, enhance treatment efficacy via augmented feedback and document the benefits of specific exercise treatment approaches. For specific muscles and movements RUSI has been found to be a valid means to assess muscle structure (morphology) and function qualitatively and quantitatively [12] [13]. 
USI evaluation through the brightness mode (B-mode) is the most common form used by physical therapists. This brief and useful guide will focus on the synthetic structure of the SIS.

\subsection{Guide for Capturing the Ultrasound Image of the Lower Trapezius and Serratus Anterior Muscles}

Focus: The alterations in scapular muscle function are involved in shoulder pain and specific motor control impairments [14] [15]. During arm elevation, the scapula upwardly rotates and posteriorly tilts while maintaining congruency to the thorax due to the regional stabilizing function of the trapezius and serratus anterior muscles [16] [17] [18]. Rehabilitative Ultrasound Imaging (RUSI) has proved to be a useful tool in identifying dysfunction of regional stabilizing muscle [19].

Ultrasound scanner and transducer: This is a real-time Ultrasound scanner in B-mode (Portable Mindray DP-20) with high resolution in which the linear array transducer (Mod. 75L38EB) with 5 to $10 \mathrm{MHz}$ (50 mm length) is set at 8.0 $\mathrm{MHz}$ for image acquisition of the serratus anterior and lower trapezius muscles [20].

Practical procedure: The imaging of the lower trapezius muscle is performed with the subject lying prone, with the head and neck in neutral alignment.

A pillow is placed under the abdomen to minimize lumbar hyperextension.

The arm of the shoulder is tested passively by movingit to 120 degrees of abduction, with the elbow extended and thumb pointing upward.

During the rest condition subjects are verbally encouraged to relax the arm tested and then an image is captured.

During the active contraction condition, subjects are verbally encouraged to relax the arm tested and then an image is captured [21].

The transducer is positionedso as to obtain the best possible image.

The landmarks for probe position are at the level of the fifthdorsal vertebra [T5], positioned by palpation.

When the T5 has been identified, the transducer is first placed centrally and horizontally over the T5 spinous process producing a bilateral image of the medial portions of the lower trapezius muscle. Then the transducer is moved laterally to the thickest part of the muscle.

An image is captured and saved to the scanner once the muscle borders are clear and parallel [22].

The subject is tested in a seated upright position without back support with knees and shoulder-width apart and feet flat on the floor (Figure 1).

The subject's arm is positioned at 120 degrees of active or passive of flexion with elbow extended and thumb facing upward.

The arm during passive flexion is supported manually by a second examiner.

The subject is asked during the active condition to keep the arm in this position.

The inferior angle of the scapula is first identified while the arm is positioned 

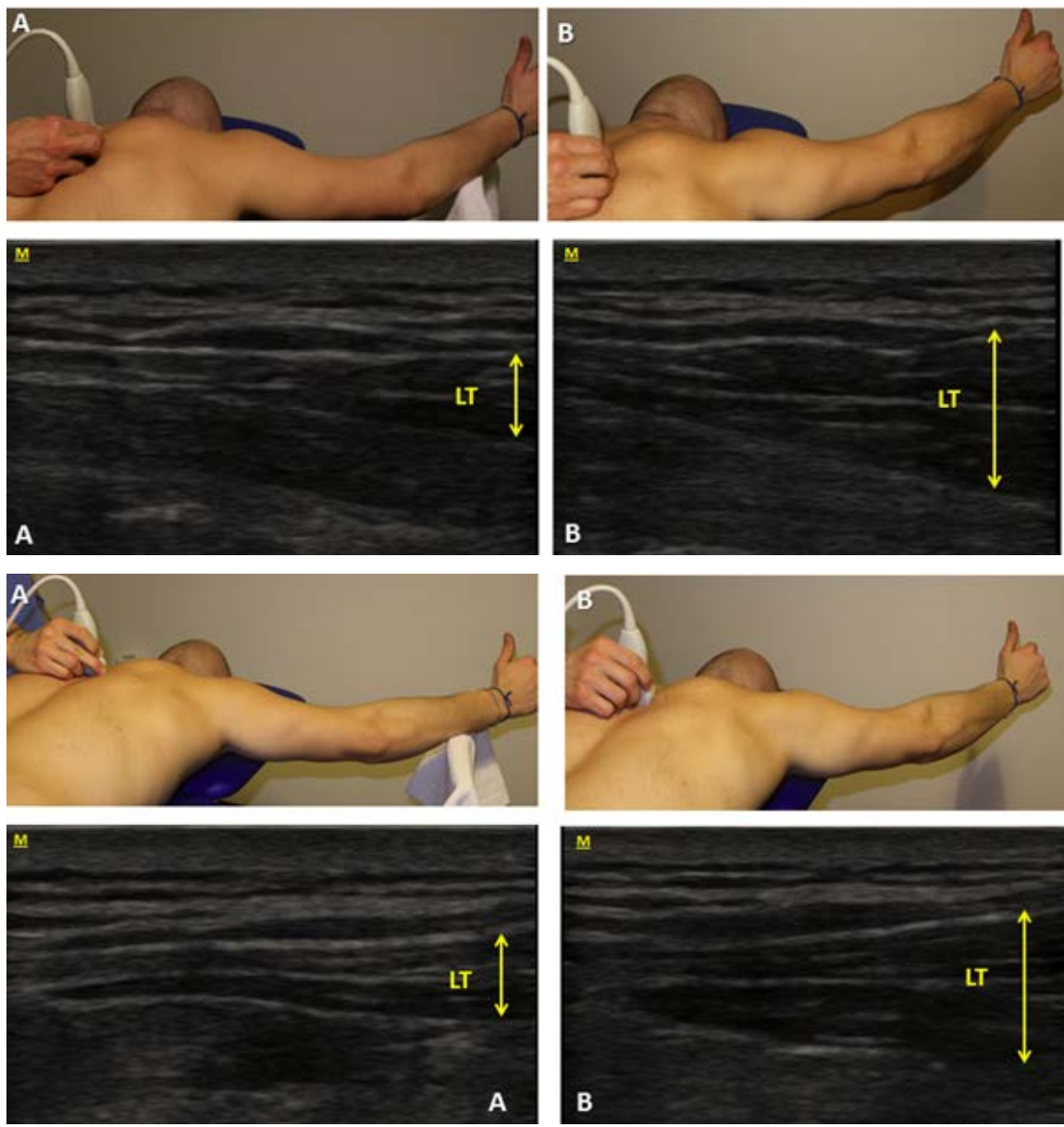

Figure 1. Longitudinal section ultrasound imaging measurement of lower trapezius (LT) at rest (A) and with contraction (B); Cross section ultrasound imaging measurement of lower trapezius (LT) at rest (A) and with contraction (B).

at 120 degrees shoulder flexion to position the US transducer.

The transducer is moved anteriorly between the latissimusdorsi and pectoralis major muscles directly over the first apparent rib angle [23] [24].

An image is captured once the hyperechoic serratus anterior muscle margins and superior rib are parallel. Images of two separate trials are captured during rest and during active contraction against gravity, (Figure 2).

\subsection{Guidefor Capturing the Ultrasound Image of the Infraspinatus Muscle}

Focus: It is hypothesized that the decreased function of the infraspinatus, as seen in SIS, contributes to the production of SIS pain [25].

Ultrasound scanner and transducer: This is a real-time Ultrasound scanner in B-mode (Portable Mindray DP-20) with high resolution. The linear array transducer (Mod. 75L38EB) with 5 to $10 \mathrm{MHz}$ (50 mm length) is set at $7.5 \mathrm{MHz}$ for image acquisition of the infraspinatus muscles [20].

Practical procedure: The subject is prone with at 90 degrees left shoulder abduction. 


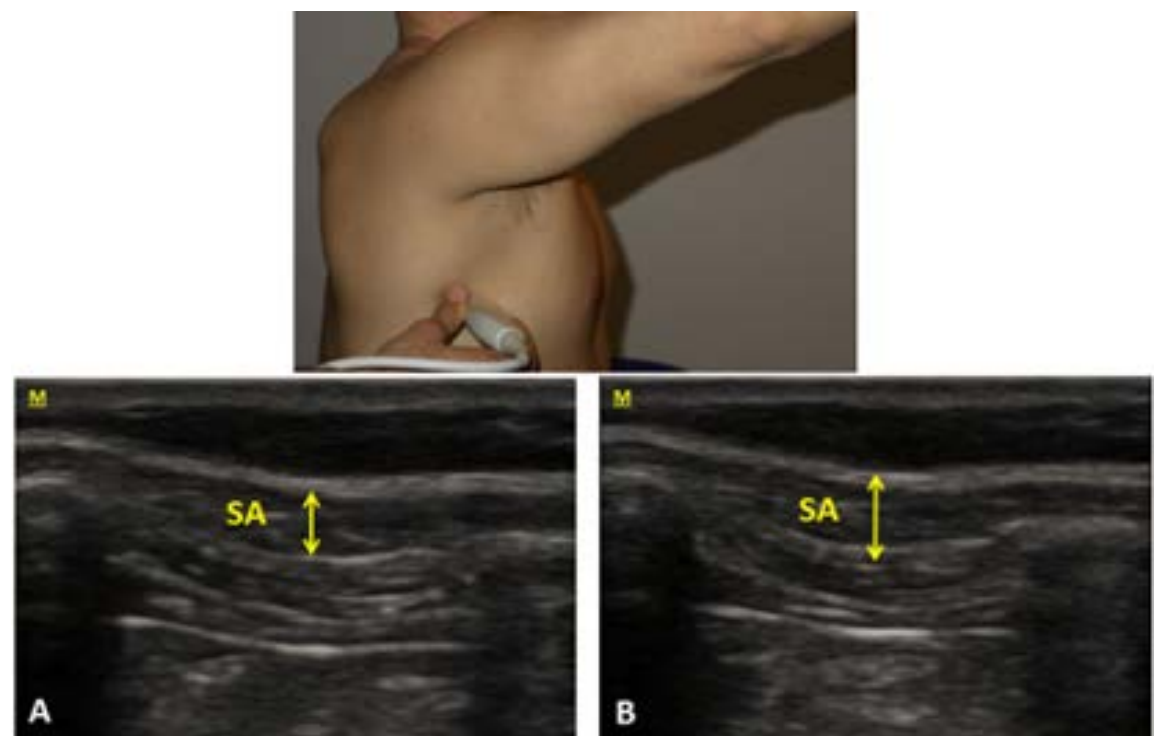

Figure 2. Longitudinal section ultrasound imaging measurement of serratus anterior (SA) at rest (A) and with contraction (B).

The upper arm subject is supported by a table while the crease of the elbow rests comfortably.

The wrist is secured to a pressure cuff, which is also secured to the table to prevent unintended movement and enable a measureable, standardized isometric contraction of the infraspinatus muscle.

The subject's head is rotated ipsilaterally so the pressure cuff gauge measuring the mmHg exerted against the cuff can be seen.

The ultrasound transducer is positioned so the superomedial border of the spine of the scapula is lined up on the left side of the ultrasound screen.

During imaging the examiner first identifies the medial border of the scapula while scanning in the transverse plane parallel with the orientation of the infraspinatus muscle fibers.

The subject is instructed to rotate the shoulder externally until a pressure of $20 \mathrm{mmHg}$ (approximately 20\% - 30\% maximal voluntary contraction) is exerted through the cuff secured to their wrist.

The pressure is maintained until an ultrasound image of the isometric contracted infraspinatus is taken [26], (Figure 3).

\subsection{Guide for Capturing the Ultrasound Image of the Acromion-Humeral Distance}

Focus: Preservation of the acromion-humeral distance (AHD) is important in to prevent impingement of the rotator cuff tendons in the sub-acromial space given that the reduced acromion-humeral distance has been associated with subacromial impingement syndrome [25].

Ultrasound scanner and transducer: This is a real-time Ultrasound scanner in B-mode (Portable Mindray DP-20) with high resolution. The linear arraytransducer (Mod. 75L38EB) with 5 to $10 \mathrm{MHz}$ (50 mm length) is set at $9.0 \mathrm{MHz}$ 

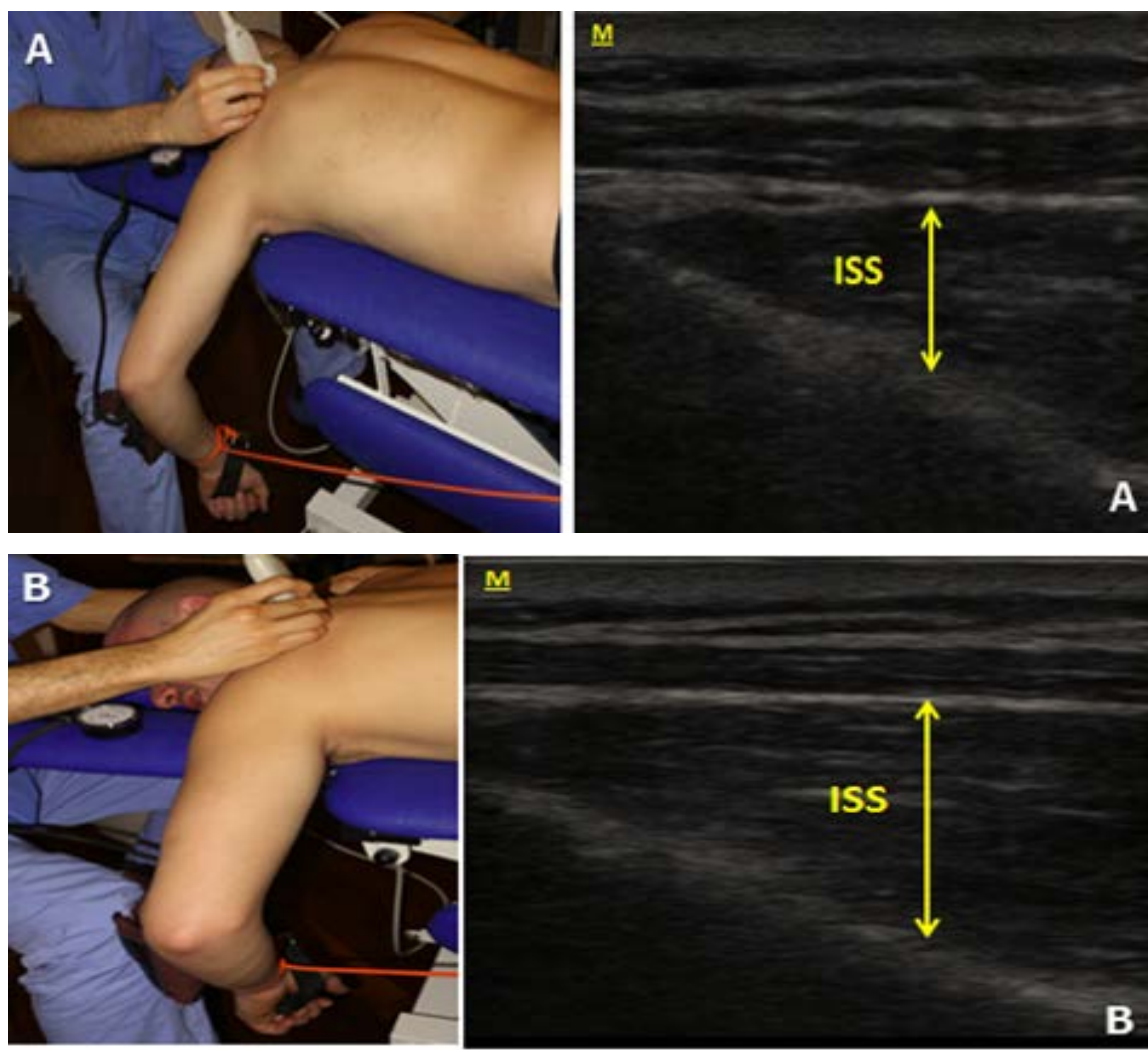

Figure 3. Longitudinal section ultrasound imaging measurement isometric infraspinatus muscle contraction procedure infraspinatus muscle (ISS) at rest (A), infraspinatus muscle contract (B).

for image acquisition of the acromion-humeral distance [20].

Practical procedure: The ultrasonographic measurement of the sub-acromial space is defined as the tangential distance between the humeral head and the edge of the acromion visible on the longitudinal sonogram as hyperechoic bony landmarks when the image is a freeze frame.

The measurement obtained therefore represents the AHD at the inlet of the sub-acromial space.

The measurements are taken with the patient sitting with the arm at $0^{\circ}$, at $45^{\circ}$ and $60^{\circ}$ of active abduction, with the elbow at $90^{\circ}$ of flexion. Because of imaging technique constraints, measurements over $60^{\circ}$ of abduction are not possible.

To maintain neutral rotation of the shoulder, the subject is instructed to maintain the hand in pronation to avoid any internal rotation. Another set of measurements it is obtained with the arm at $45^{\circ}$ and $60^{\circ}$ abduction.

The ultrasonography measurements of AHD are done with the probe positioned on the lateral surface of the shoulder along the longitudinal axis of the humeruswithin the first $2 \mathrm{~cm}$ at the anterior part of the acromion.

The AHD is measured at the anterior part of the acromial arch and at $1 \mathrm{~cm}$ behind this first measurement. For each position on the acromion, 2 measurements with less than $10 \%$ variation are made, and the mean AHD is calculated [27], (Figure 4). 

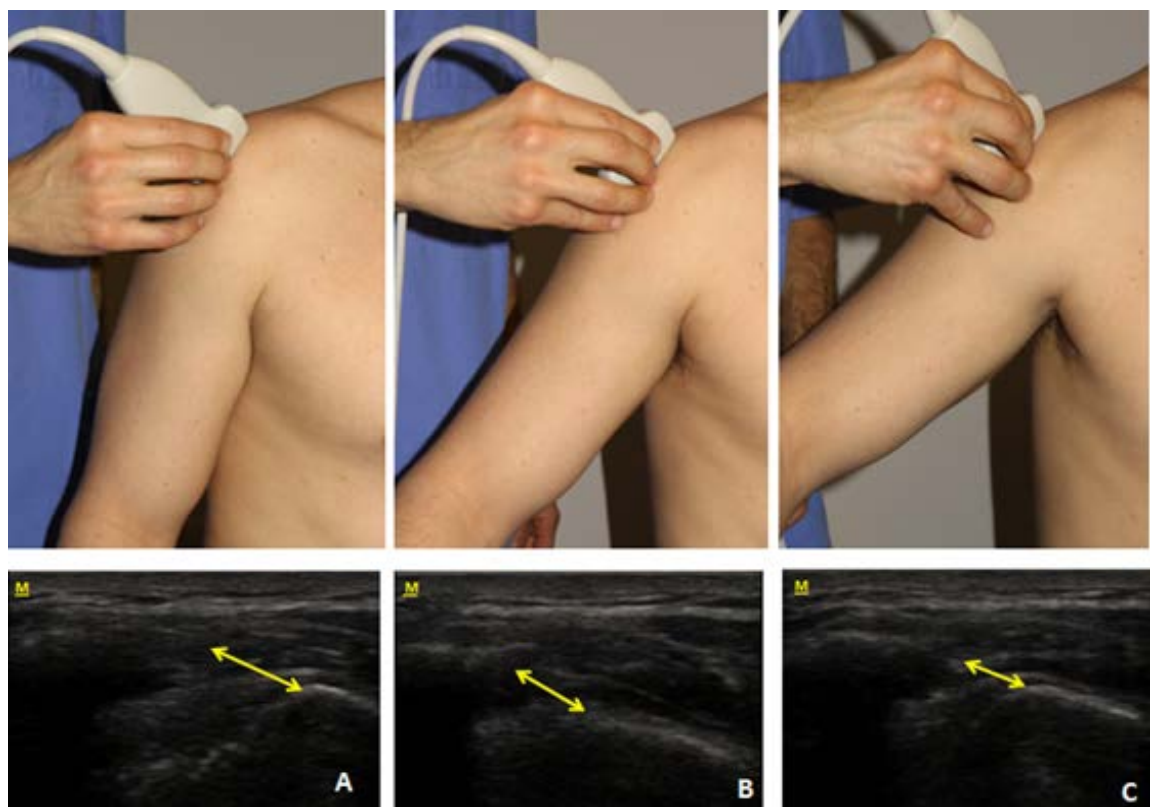

Figure 4. The ultrasound transducer was placed in the coronal plane parallel with the longitudinal axis of the humerus. The ultrasound transducer was maintain in three positions the abduction $0^{\circ}, 45^{\circ}, 60^{\circ}(\mathrm{A})$; (B); (C). The shortest tangential measurement between the hyper echoic landmarks of the most superior aspect of the humerus and acromion (the acromion-humeral distance) is shown on the ultrasound image.

\subsection{Guide for Capturing the Ultrasound Image of the Sub-Coracoid Bursae}

Focus: This is an effective way to evaluate subcoracoid impingement syndrome with bursitis, and help in providing a differential diagnosis of anterior shoulder pain. It can be useful in the evaluation because of its high resolution capacity and the use of dynamic maneuvers [28].

Ultrasound scanner and transducer: This is a real-time Ultrasound scanner in B-mode (Portable Mindray DP-20) with high resolution. The linear array transducer (Mod. 75L38EB) with 5 to $10 \mathrm{MHz}(50 \mathrm{~mm}$ length) is set at $9.0 \mathrm{MHz}$ for image acquisition of the SubcoracoidBursae [20]-[28].

Practical procedure: The patient in the sitting position: Rotate the arm externally fixing the elbow on the iliac crest to show the subscapularis tendon and its insertion on the lesser tuberosity (slight supination of the hand may be helpful) and the long-axis view of the isoechoic heterogeneous subcoracoid bursa to the subscapularis tendon on external rotation of the shoulder [28], (Figure 5).

\subsection{Guide for Capturing the Ultrasound Image of the Subscapularis Tendon}

Focus: This is an effective way to evaluate subcoracoid impingement syndrome with tendinopathy, andaid a differential diagnosis of anterior shoulder pain. It can be useful in the evaluation because of its high resolution capacity and the use of dynamic maneuvers. It is important to recognize and treat subscapularis tendon tears because this muscle plays an essential role in the stability of glenohumeral joint [29]. 

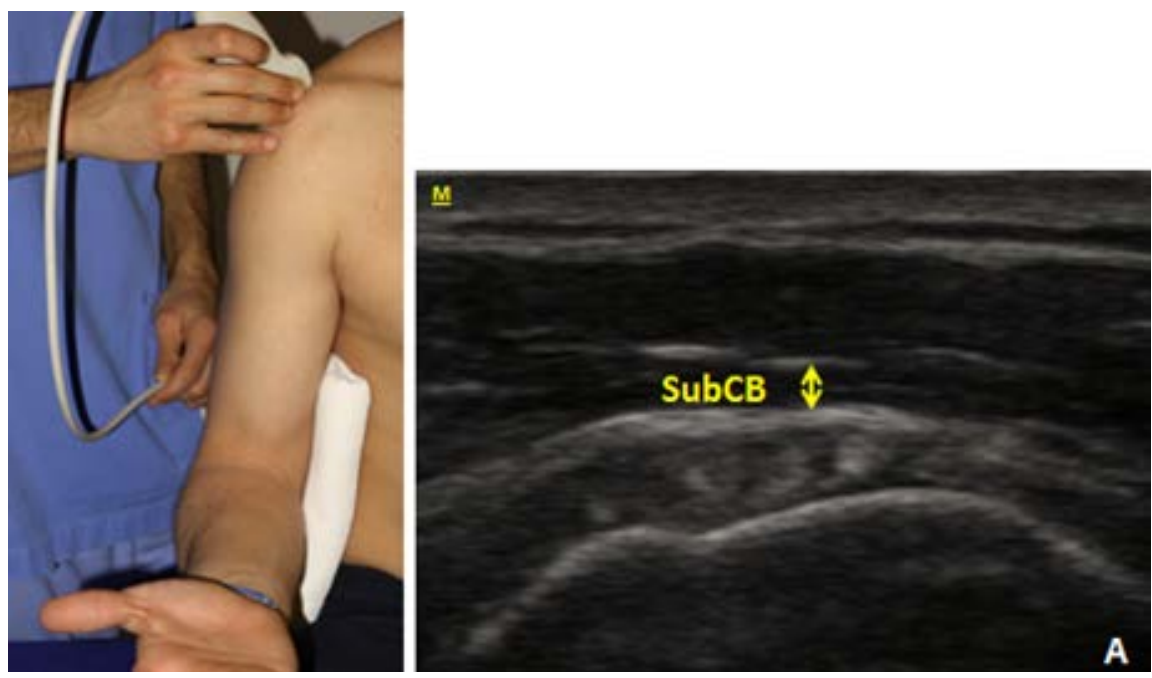

Figure 5. Longitudinal section ultrasound imaging measurement of Sub-Coracoid Bursae (SubCB), (A).

Ultrasound scanner and transducer: This is a real-time ultrasound scanner in B-mode (Portable Mindray DP-20) with high resolution linear array transducer (Mod. 75L38EB) with 5 to $10 \mathrm{MHz}$ (50 mm length).The frequency linear array transducer set at $8.0 \mathrm{MHz}$ was used to capture greyscale B-mode images for the tendon Subscapularis [20].

This is a real-time Ultrasound scanner in B-mode (Portable Mindray DP-20) with high resolution. The linear array transducer (Mod. 75L38EB) with 5 to 10 $\mathrm{MHz}$ (50 mm length) is set at $8.0 \mathrm{MHz}$ for image acquisition of the Subscapularis Tendon [20].

Practical procedure: Rotate the arm externally holding the elbow on the iliac crest to show the subscapularis tendon and its insertion on the lesser tuberosity (slight supination of the hand may be helpful to neutralize the tendency to lift and abduct the elbow from the lateral chest wall).

This tendon is evaluated in its two planes (transverse planes and sagittal planes) during passive external and internal rotation with hanging arm. Sweep the transducer up and down over the subscapularis until its full width is shown ([30]), (Figure 6).

\subsection{Guide for Capturing the Ultrasound Image of the Supraspinatus Tendon}

Focus: This is an effective way to evaluate subcoracoid impingement syndrome with tendinopathy andaid a differential diagnosis of anterior shoulder pain. It can be useful in the evaluation because of its high resolution capacity and the use of dynamic maneuvers [25].

Ultrasound scanner and transducer: This is a real-time Ultrasound scanner in B-mode (Portable Mindray DP-20) with high resolution. The linear array transducer (Mod. 75L38EB) with 5 to $10 \mathrm{MHz}$ (50 mm length) is set at $8.0 \mathrm{MHz}$ for image acquisition of the Supraspinatus tendon [20]. 


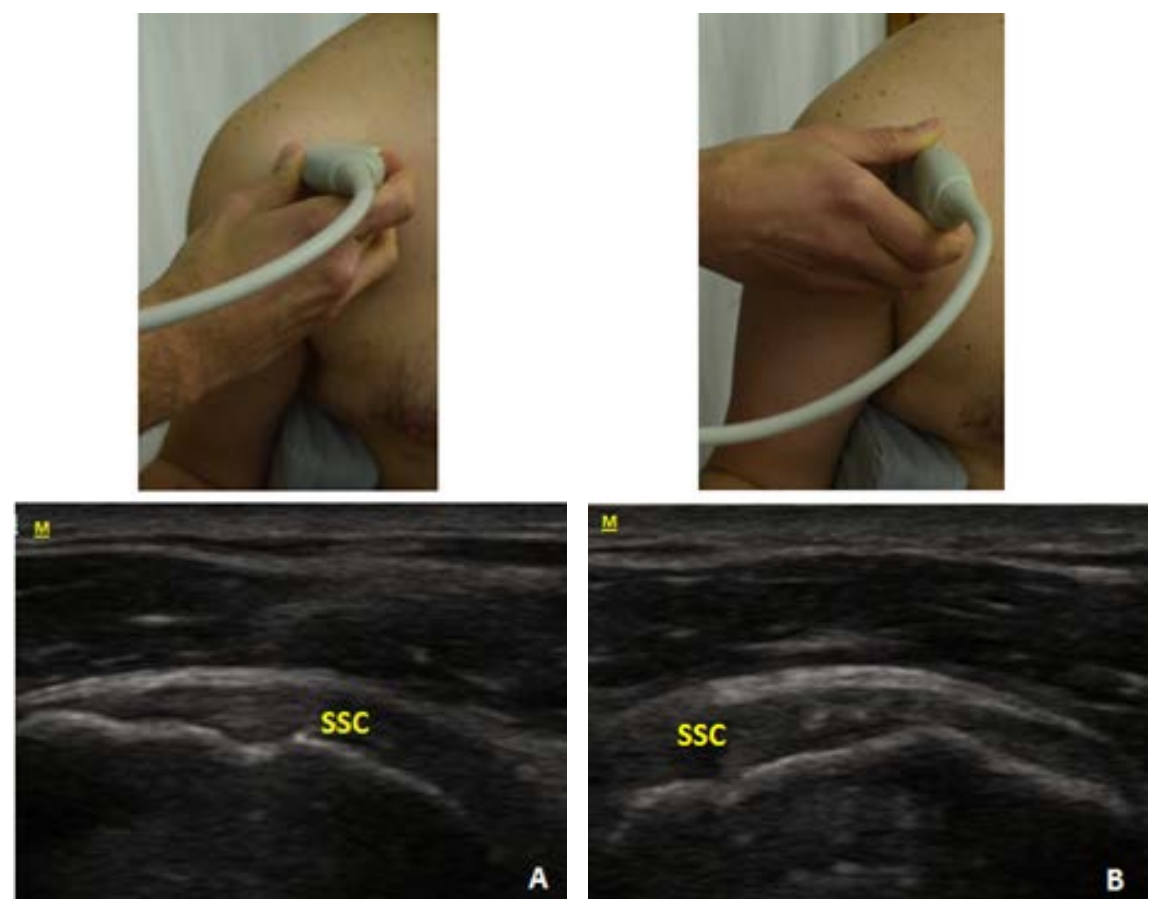

Figure 6. Longitudinal section ultrasound imaging measurement of Subscapularis tendon (SSC), (A); Cross section ultrasound imaging measurement of Subscapularis tendon (SSC), (B).

Practical procedure: The arm of the patient is positioned lacing the palmar side of the hand on the superior aspect of the iliac wing with the elbow flexed and directed posteriorly.

The supraspinatus tendon is evaluated along its long and short axis.

The reference image is the intraarticular portion of the biceps as a landmark to obtain proper transducer orientation for imaging the supraspinatus.

Remember totilt the transducer slightly in the area overlying the tendon insertion to avoid anisotropy.

When looking for the supraspinatus on the shortaxis, the normal cuff must have almost the same thickness from the biceps tendon landmark for $2 \mathrm{~cm}$ (backwards: from this point backwards the tendon seen is the infraspinatus), the supraspinatus tendon must be assessed along its long and shortaxes [30], (Figure 7).

\subsection{Guide for Capturing the Ultrasound Image of the Infraspinatus Tendon}

Focus: Infraspinatus tendinopathy is usually caused by overuse and the pain is felt at the back of the shoulder when performing the outward movement against some resistance and on lifting the arm up as in waving to someone, when a pinch of pain may be felt at shoulder height [31].

Ultrasound scanner andtransducer: This is a real-time Ultrasound scanner in B-mode (Portable Mindray DP-20) with high resolution. The linear array transducer (Mod. 75L38EB) with 5 to $10 \mathrm{MHz}$ (50 mm length) is set at $8.0 \mathrm{MHz}$ 

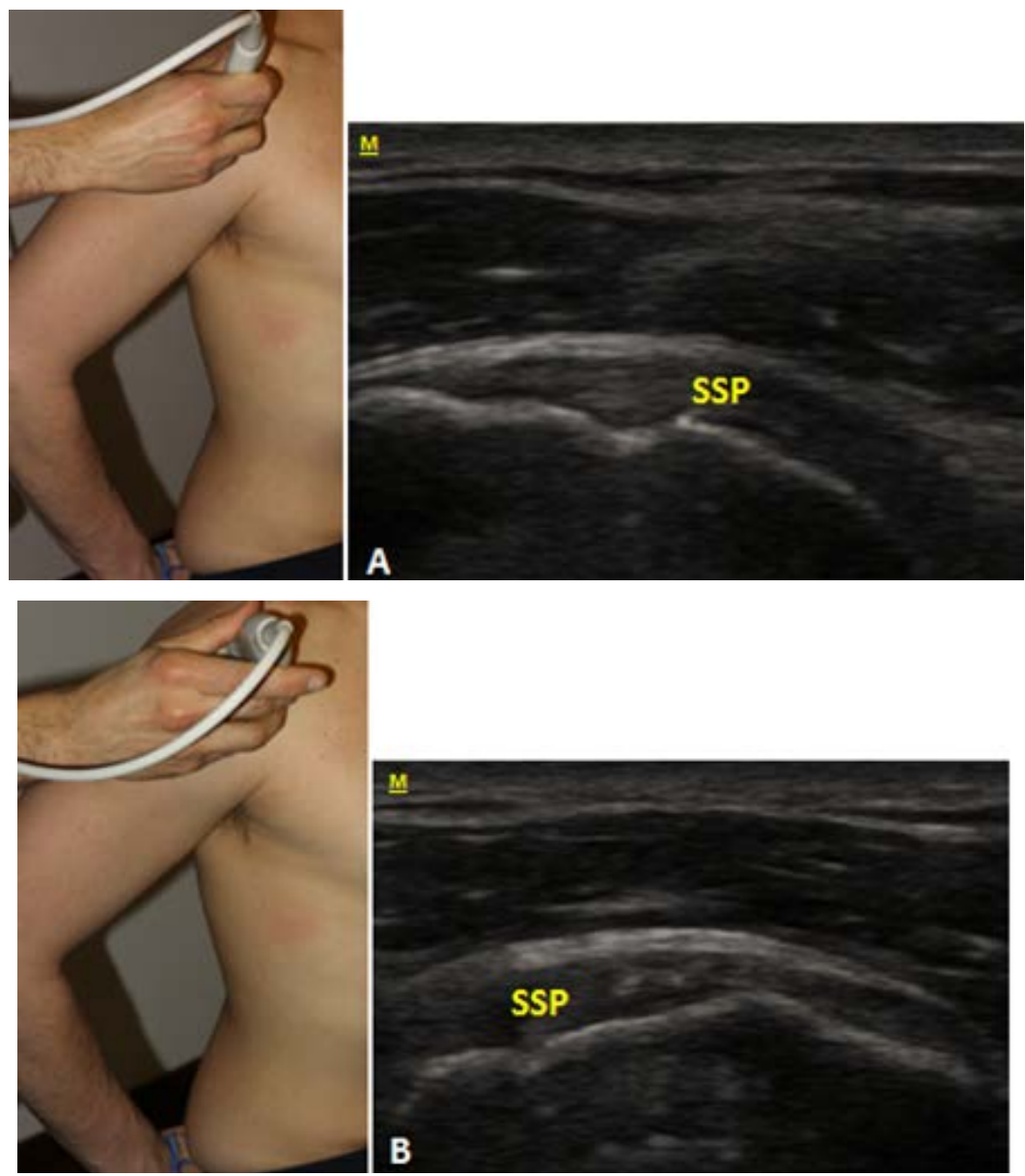

Figure 7. Longitudinal section ultrasound imaging measurement of Supraspinatus tendon (SSP), (A); Cross section ultrasound imaging measurement of Supraspinatus tendon (SSP), (B).

for image acquisition of the Infraspinatus tendon [20].

Practical procedure: The transducer is positioned over the posterior aspect of the glenohumeral joint with the hand on the opposite shoulder and increase in depth to include the structures of the posterior fossa within the field-of view of the US image.

Use the spine of the scapula as the landmark to distinguish the supraspinous fossa (transducer shifted up) from the infraspinous fossa (transducer shifted down) on sagittal planes.

The spine of the shoulder is used as the landmark to distinguish the supraspinous fossa (transducer shifted up) from the infraspinous fossa (transducer shifted down) on sagittal planes.

The infraspinatus and teres minor muscles are seen as single structures filling in the infraspinous fossa to the depth of the deltoid.

After scanning the muscles, sweep the transducer toward the greater tuberosity on sagittal planes.

The two tendons can be seen as individual structures rising from the respective muscle [31], (Figure 8). 

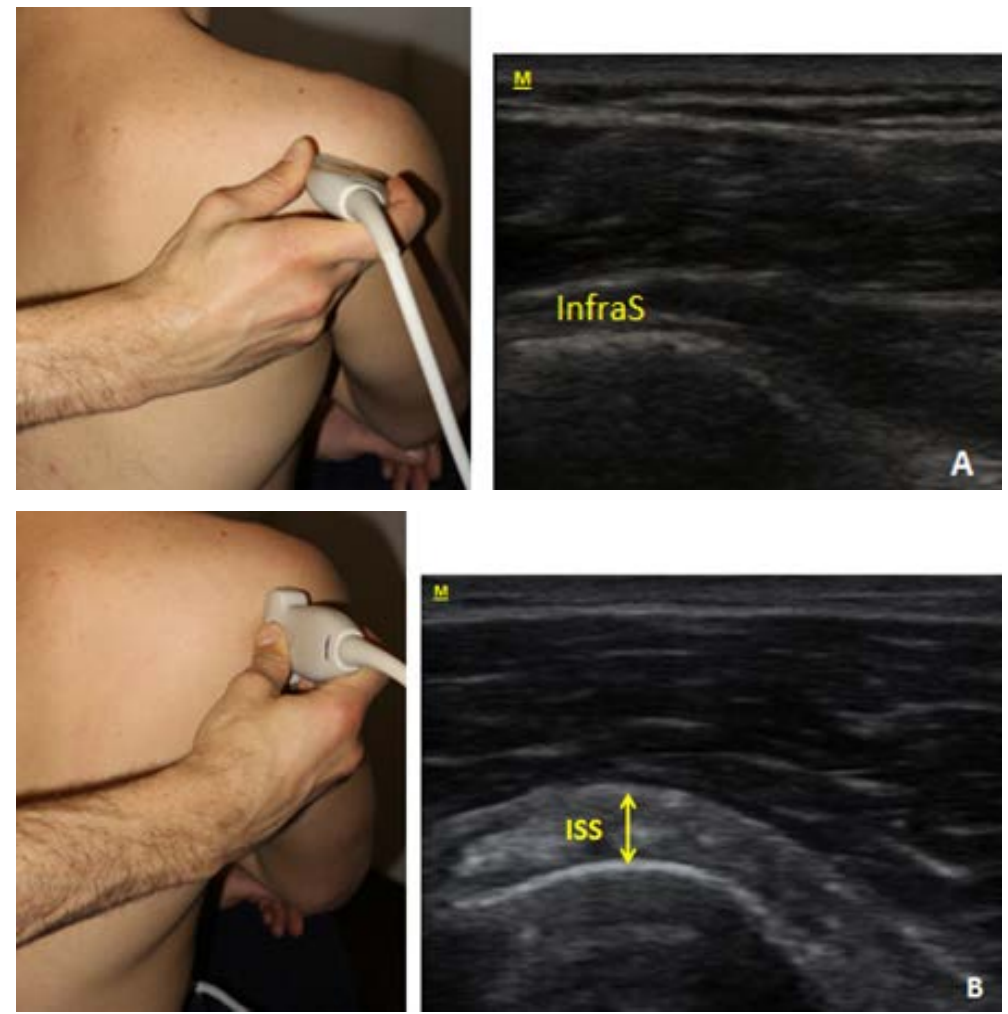

Figure 8. Longitudinal section ultrasound imaging measurement of Infraspinatus tendon (ISS), (A); Cross section ultrasound imaging measurement of Infraspinatus tendon (ISS), (B).

\subsection{Guide for Capturing the Ultrasound Image of the Subscapularis Muscle}

Focus: The subscapularis muscle is the largest and strongest muscle of the rotator cuff.

The subscapularis muscle allows internal rotation of the humerus, provides anterior stability of the shoulder and is involved in balancing force couples of the glenohumeral joint. It provides 50\% the of total cuff strength.

Clinical presentation is usually characterized by a history of pain, typically located anteriorly, and difficulty in lifting movements across the chest, or twisting inwards, that hinders daily activities.

The special tests for diagnosis of subscapularis tears include the lift-off, bellypress, and bear-hug tests.

The imaging of the subscapularis tendon may involve plain radiography, magnetic resonance and ultrasound scanning [32].

Ultrasound scanner and transducer: This is a real-time Ultrasound scanner in B-mode (Portable Mindray DP-20) with high resolution. The linear array transducer (Mod. 75L38EB) with 5 to $10 \mathrm{MHz}$ (50 mm length) is set at $8.0 \mathrm{MHz}$ for image acquisition of the Subscapularis muscle [20].

Practical procedure: For the procedure involving the subscapularis muscle, the patient is supine with the shoulder abducted, elbow flexed, hand supinated, and scapula rotated as upwardly as possible to expose the subscapularis. 
In this position, only the teres major muscle is located above the subscapularis. The pectoralis major and latissimusdorsi are not placed in the assessment window.

The region is scanned and a transverse plane is obtained to visualize the target muscle.

The lateral border of the scapula is identified by ultrasonography [32], (Figure 9).

\subsection{Guide for Capturing the Ultrasound image of the Upper Trapezius Muscle}

Focus: The alterations in scapular muscle function that are proposed contribute to abnormal scapular motion and shoulder pain [14].

Ultrasound scanner and transducer: This is a real-time Ultrasound scanner in B-mode (Portable Mindray DP-20) with high resolution. The linear array transducer (Mod. 75L38EB) with 5 to $10 \mathrm{MHz}(50 \mathrm{~mm}$ length) is set at $8.0 \mathrm{MHz}$ for image acquisition of the Middle Trapezius muscle [20].

Practical procedure: The subject is seated upright on the edge of a plinth with the knees and hips at 90 degrees and their feet flat on the floor.

The upper arms by the sides, elbows are flexed to 90 degrees and forearms are pronated and supported on a pillow, so that the palms face the floor.

In this position, transducer placement is determined by drawing a line between the posterolateral edge of the acromion and the spinous process of the T1 vertebra, (using a pencil).

The distance between the two bony prominences is measured, divided by three, and the transducer isplaced horizontally over the middle third.
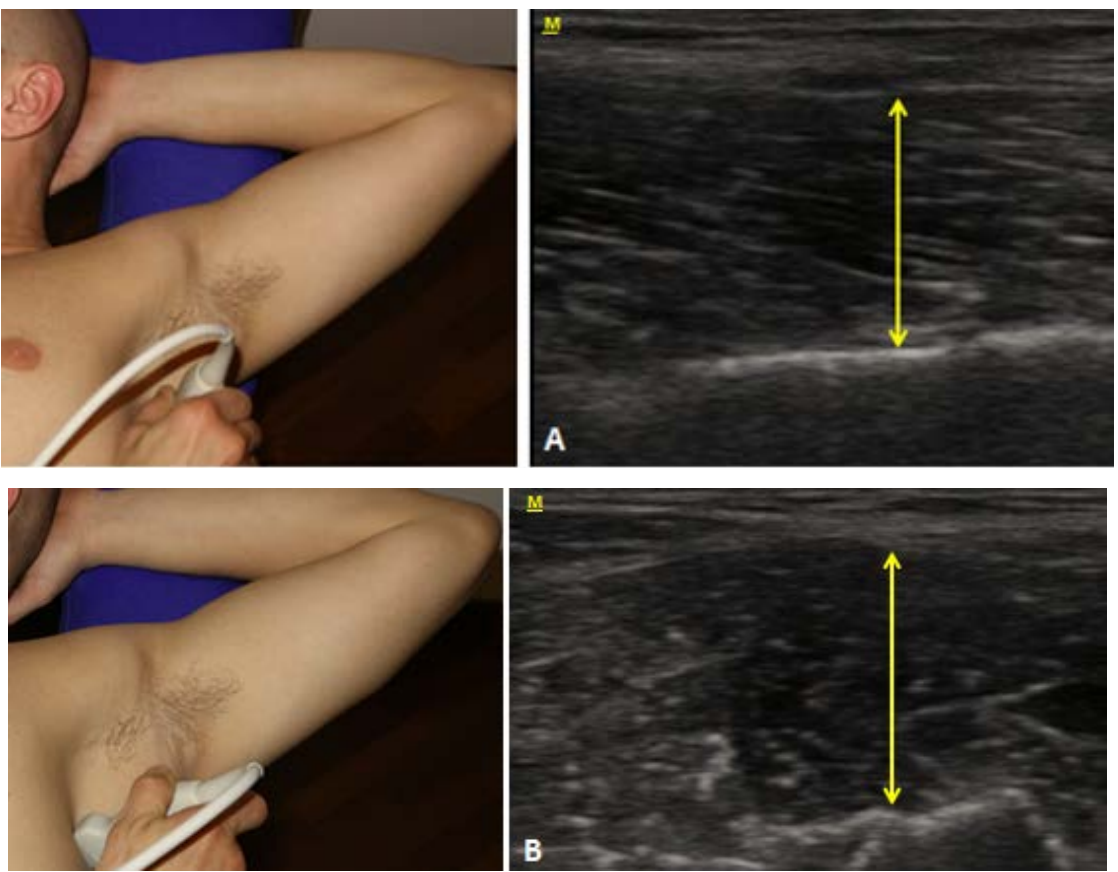

Figure 9. Longitudinal section ultrasound imaging measurement of Subscapularis (SSC), (A); Cross section ultrasound imaging measurement of Subscapularis (SSC), (B). 
The transducer is tilted in an anterior and inferior direction, in line with the curvature of the shoulder, until the echogenic fascial borders of the middle trapezius muscle are displayedeasily on the screen [33], (Figure 10).

\subsection{Guide for Capturing the Ultrasound Image of the Supraspinatus Muscle}

Focus: In cases of shoulder pain most tears occur in the supraspinatus muscle and tendon. For this reason it is very important to study the supraspinatus muscle morphology [33].

Ultrasound scanner and transducer: This is a real-time Ultrasound scanner in B-mode (Portable Mindray DP-20) with high resolution. The linear array transducer (Mod. 75L38EB) with 5 to $10 \mathrm{MHz}$ (50 mm length) is set at $7.5 \mathrm{MHz}$ for image acquisition of the Middle Trapezius muscle [30].

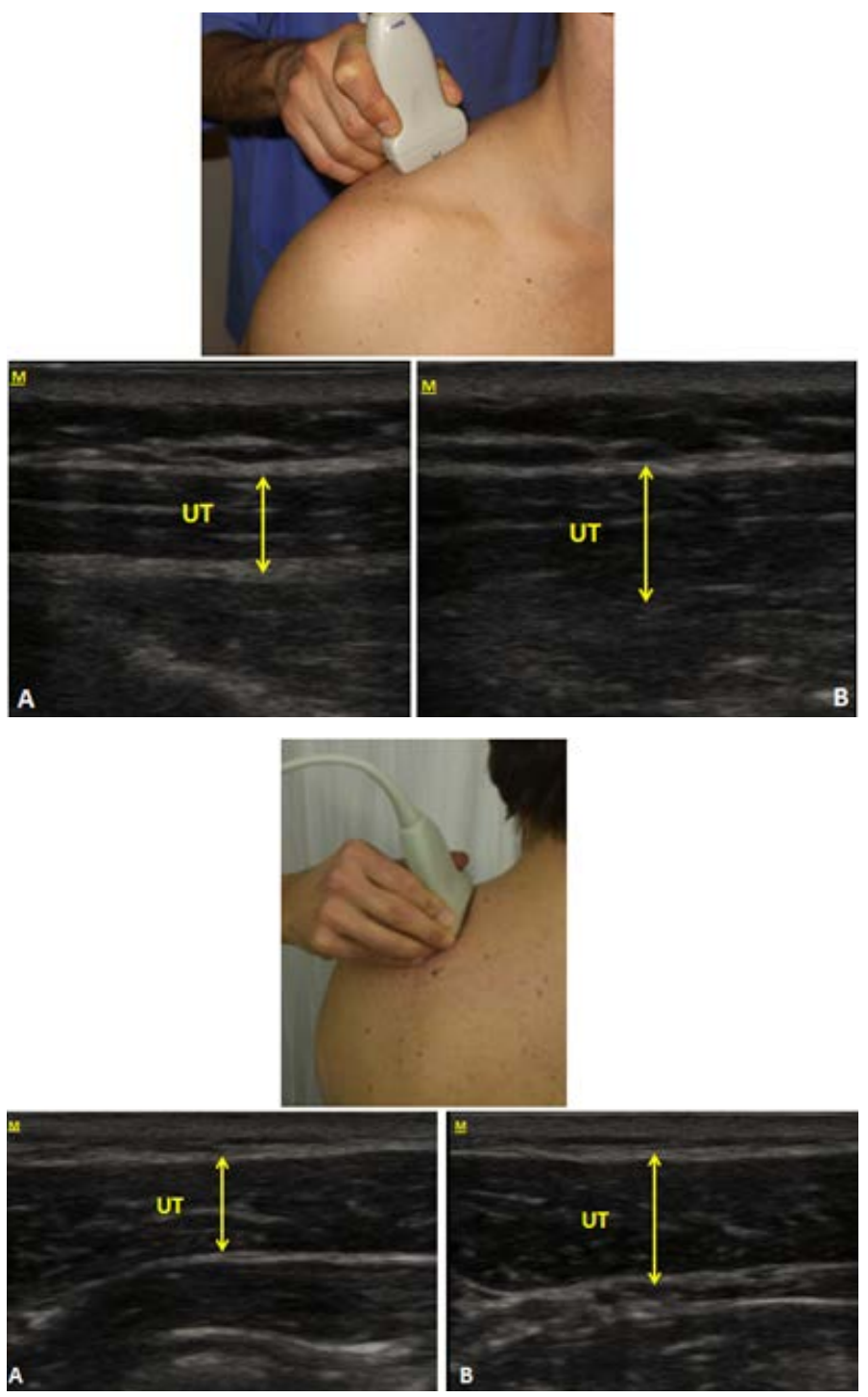

Figure 10. Longitudinal section ultrasound imaging measurement of upper trapezius (UT) at rest (A) and with contraction (B); Cross section ultrasound imaging measurement of upper trapezius (UT) at rest (A) and with contraction (B). 
Practicalprocedure: The subject is seated on a chair with arm lying along the side; the palm is facing the body, and the head and neck is in a neutral position.

Thickness and cross-sectional area is measured for each muscle.

To obtain the muscle thickness, the spine of the scapula is identified by palpation and the probe is placed horizontally and superiorly to it.

The supraspinatus isdisplayed at the bottom of the B-mode image and it is easily identifiable because of its triangular shape.

The muscle thickness is measured at $20.0 \mathrm{~mm}$ from the angle formed by the superior muscular fascia of the supraspinatus and the medial part of the supraspinous fossa [30], (Figure 11).

\subsection{Guide for Capturing the Ultrasound Image of the Teres Minor Muscle}

Focus: The teres minor muscle tear may jeopardize glenohumeral joint stability by disrupting the rotator cuff force couple, leading to limited ability to elevate above the horizontal.

An intact teres minor makes an important contribution to shoulder function in patients with large or massive tears of the rotator cuff; it contributes enough power to externally rotate the abducted arm, helps to maintain the ability to perform important daily activities and reduces the symptoms of rotator cuff tears [34].

Ultrasound scanner and transducer: This is a real-time Ultrasound scanner in B-mode (Portable Mindray DP-20) with high resolution. The linear array transducer (Mod. 75L38EB) with 5 to $10 \mathrm{MHz}$ (50 mm length) is set at $7.5 \mathrm{MHz}$ for image acquisition of the Muscle Teres Minor [20].

Practicalprocedure: The longitudinal view of the teres minor insertion is obtained with the transducer placed parallel to the direction of the teres minor
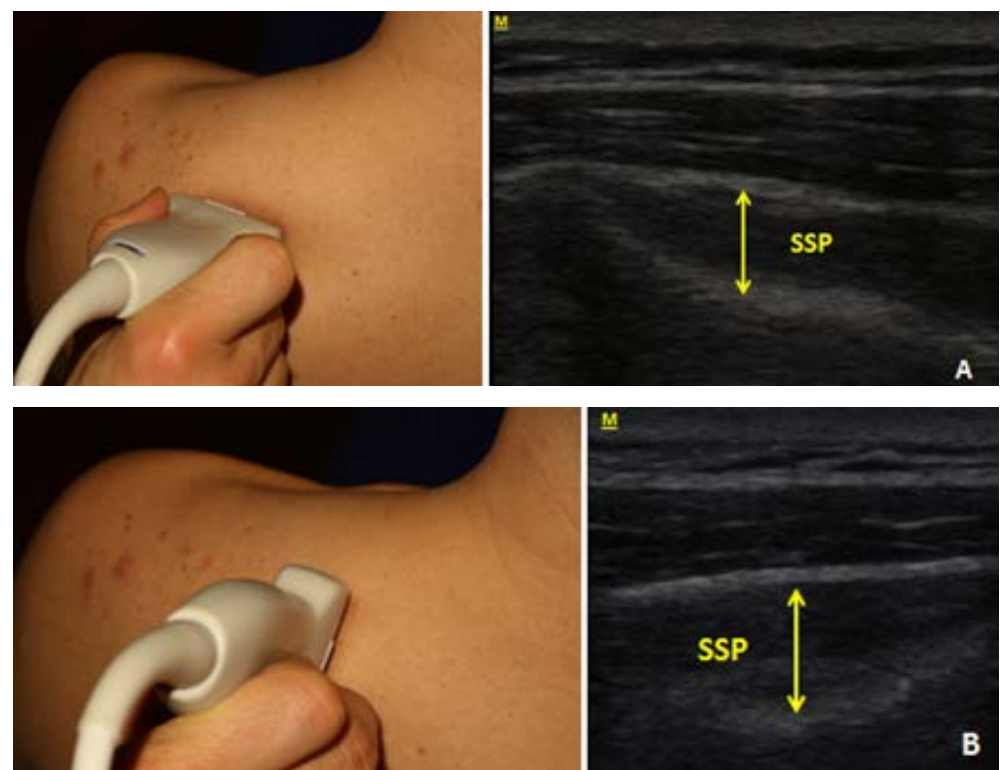

Figure 11. Longitudinal section ultrasound imaging of supraspinatus muscle (SSP), (A); Cross section ultrasound imaging of supraspinatus muscle (SSP), (B). 
muscle fibers at the level of the posterior glenohumeral joint line (transducer placed $2 \mathrm{~cm}$ lateral and $1 \mathrm{~cm}$ anterior to the posterolateral corner of the acromion).

Since the teres minor originates from the middle portion of the lateral border of the scapula and inserts onto the inferior aspect of the posterior greater tuberosity, the direction of the teres minor muscle fibers is slightly oblique from inferomedial to superolateral.

Therefore, the transducer is oriented in an oblique plane of approximately $30^{\circ}$ to the scapular spine.

The transverse view of the teres minor is obtained with the transducer placed perpendicular to the direction of the muscle fibers at the level of the spine of the scapula [34], (Figure 12).

\section{Conclusions}

RUSI can be useful for identifying muscle dysfunction in patients with SIS and therefore, may be useful in predicting individuals that respond to certain interventions or who are at risk for developing chronicity.

Ultrasonography is a rapid, non-invasive, and inexpensive adjunct to functional examination in patients with SIS.

Designing a well-thought-out, collaborative research agenda across multiple clinical research networks would be a prudent next step to answer important questions such as the standardization of RUSI.
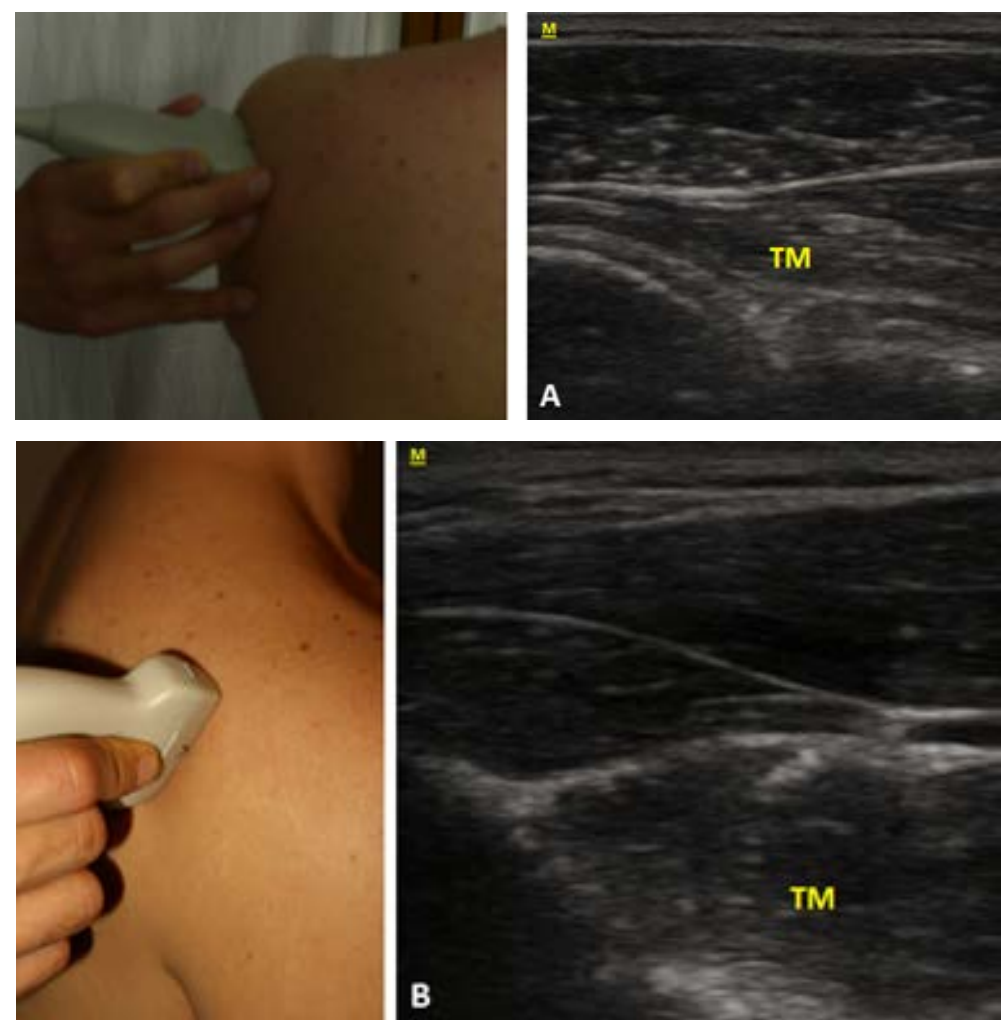

Figure 12. Longitudinal section ultrasound imaging measurement of Teres Minor (TM), (A); Cross section ultrasound imaging measurement of Teres Minor (TM), (B). 
It is believed that RUSI is a tool that can help physiotherapists in evaluation and treatment in the improvement of the damage.

Additionally, RUSI is used in basic, applied, and clinical rehabilitative research to inform clinical practice.

If validated in future research, RUSI may allow for more objective quantifications of muscle impairments and be a useful adjunct to physical examination in patients with SIS.

The direction of research in the reliability and the application of RUSI is promising and is attempting to improve the methodological aspects of the studies as well as the clinical impact as a classification tool, thus providing accurate strategies for the implementation of rehabilitation practice.

Currently, the international community is developing education and safety guidelines in accordance with the World Federation for Ultrasound in Medicine and Biology (WFUMB), [10].

The financial crisis has caused a severe limitation of resources for the public health service and rehabilitation.

The proposed diagnosis and integrated treatment in rehabilitation, which involves the introduction of new therapeutic models and evaluation alongside orthodox models, could lead to a reduction in costs through the addition of a RUSI model.

\section{Acknowledgements}

The authors would like to thank Dr. Cristina Ferroni of Tecnomedical Ltd. who loaned the portable ultrasound unit used in this study.

\section{References}

[1] Feleus, A., Bierma-Zeinstra, S., Miedema, H., Bernsen, R., Verhaar, J. and Koes, B. (2008) Incidence of Non-Traumatic Complaints of Arm, Neck and Shoulder in General Practice. Manual Therapy, 13, 426-433.

[2] Koester, M., George, M. and Kuhn, J. (2005) Shoulder Impingement Syndrome. American Journal of Medicine, 118, 452-455.

[3] Escamilla, R., Hooks, T. and Wilk, K. (2014) Optimal Management of Shoulder Impingement Syndrome. Open Access Journal of Sports Medicine, 5, 13-24. https://doi.org/10.2147/OAJSM.S36646

[4] Page, P. (2011) Shoulder Muscle Imbalance and Subacromial Impingement Syndrome in Overhead Athletes. International Journal of Sports Physical Therapy, 6, 51-58.

[5] Hakimeh, A., Azadeh, S., Esmail, E., Asghar, R. and Farrokh, N. (2016) Ultrasonography for the Assessment of the Upper Trapezius Properties in Healthy Females: A Reliability Study. Muscles Ligaments Tendons Journal, 6, 167-172.

[6] Christian, K., Toni, L., Jochen, S. and Philip, K. (2015) Interrater Reliability of the Modified Scapular Assistance Test with and without Handheld Weights. Manual Therapy, 20, 868-874.

[7] Hides, J.A., Richardson, C.A., Jull, G.A. and Davies, S. (1995) Ultrasound Imaging in Rehabilitation. Australian Journal of Physiotherapy, 41, 187-193.

[8] Stokes, M., Hides, J. and Nassiri, D.K. (2012) Musculoskeletal Ultrasound Imaging: 
Diagnostic and Treatment Aid in Rehabilitation. International Journal of Sports Physical Therapy, 7, 342-349.

[9] Whittaker, J.L., Teyhen, D.S., Elliott, J.M., et al. (2007) Rehabilitative Ultrasound Imaging: Understanding the Technology and Its Applications. Journal of Orthopaedic \& Sports Physical Therapy, 37, 434-449. https://doi.org/10.2519/jospt.2011.3682

[10] Teyhen, D. (2006) Rehabilitative Ultrasound Imaging Symposium. Journal of Orthopaedic \& Sports Physical Therapy, 36, A1-A17. https://doi.org/10.2519/jospt.2011.3682

[11] Whittaker, J.L. and Stokes, M. (2011) Ultrasound Imaging and Muscle Function. Journal of Orthopaedic \& Sports Physical Therapy, 41, 572-580. https://doi.org/10.2519/jospt.2011.3682

[12] Koppenhaver, S.L., Hebert, J.J., Parent, E.C. and Fritz, J.M. (2009) Rehabilitative Ultrasound Imaging Is a Valid Measure of Trunk Muscle Size and Activation during Most Isometric Submaximal Contractions: A Systematic Review. Australian Journal of Physiotherapy, 55, 153e69.

[13] Henry, S.M. and Teyhen, D.S. (2007) Ultrasound Imaging as a Feedback Tool in the Rehabilitation of Trunk Muscle Dysfunction for People with Low Back Pain. Journal of Orthopaedic \& Sports Physical Therapy, 37, 627e34.

https://doi.org/10.2519/jospt.2007.2555

[14] Ludewig, P.M. and Jonathan, F.R. (2009) The Association of Scapular Kinematics and Glenohumeral Joint Pathologies. Journal of Orthopaedic \& Sports Physical Therapy, 39, 90-104. https://doi.org/10.2519/jospt.2009.2808

[15] Struyf, F., Lluch, E., Falla, D., Meeus, M., Noten, S. and Nijs, J. (2015) Influence of Shoulder Pain on Muscle Function: Implications for the Assessment and Therapy of Shoulder Disorders. European Journal of Applied Physiology, 115, 225-234. https://doi.org/10.1007/s00421-014-3059-7

[16] Karduna, A.R., McClure, P.W., Michener, L.A. and Sennett, B. (2001) Dynamic Measurements of Three-Dimensional Scapular Kinematics: A Validation Study. Journal of Biomechanical Engineering, 123, 184-190. https://doi.org/10.1115/1.1351892

[17] Ebaugh, D.D., McClure, P.W. and Karduna, A.R. (2005) Three-Dimensional Scapulothoracic Motion during Active and Passive Arm Elevation. Clinical Biomechanics, 20, 700-709.

[18] Lunden, J.B., Braman, J.P., Laprade, R.F. and Ludewig, P.M. (2010) Shoulder Kinematics during the Wall Push-Up plus Exercise. Journal of Shoulder and Elbow Surgery, 19, 216-223.

[19] Seitz, A.L., Baxter, C.J. and Benya, K. (2015) Muscle Thickness Measurements of the Lower Trapezius with Rehabilitative Ultrasound Imaging Are Confounded by Scapular Dyskinesis. Manual Therapy, 20, 558e563.

[20] Mindray Bio-Medical Eletronics Co., LTD, Midray Building, Keji 12th Road South, High-Tech Industrial Park, Nanshan, Shenzhen 518057, P.R. China.

www.mindray.com

[21] Ekstrom, R.A., Soderberg, G.L. and Donatelli, R.A. (2005) Normalization Procedures Using Maximum Voluntary Isometric Contractions for the Serratus Anterior and Trapezius Muscles during Surface EMG Analysis. Journal of Electromyography and Kinesiology, 15, 418-428.

[22] O’Sullivan, C., Bentman, S., Bennett, K. and Stokes, M. (2007) Rehabilitative Ultrasound Imaging of the Lower Trapezius Muscle: Technical Description and Reliability. Journal of Orthopaedic \& Sports Physical Therapy, 37, 620-626.

https://doi.org/10.2519/jospt.2007.2446 
[23] Day, J.M. and Uhl, T. (2013) Thickness of the Lower Trapezius and Serratus Anterior Using Ultrasound Imaging during a Repeated Arm Lifting Task. Manual Therapy, 18, 588-593.

[24] Talbott, N.R. and Witt, D.W. (2013) Ultrasound Imaging of the Serratus Anterior Muscle at Rest and during Contraction. Clinical Physiology and Functional Imaging, 33, 192-200. https://doi.org/10.1111/cpf.12012

[25] Koppenhaver, S., Harris, D., Harris, A., O’Connor, E., Dummar, M., Croy. T., Walker, M. and Flynn, T. (2015) The Reliability of Rehabilitative Ultrasound Imaging in the Measurement of Infraspinatus Muscle Function in the Symptomatic and Asymptomatic Shoulders of Patients with Unilateral Shoulder Impingement Syndrome. International Journal of Sports Physical Therapy, 10, 128-135.

[26] Desmeules, F., Minville, L., Riederer, B., Côté, C.H. and Frémont, P. (2004) Acromio-Humeral Distance Variation Measured by Ultrasonography and Its Association with the Outcome of Rehabilitation for Shoulder Impingement Syndrome. Clinical Journal of Sport Medicine, 14, 197-205. https://doi.org/10.1097/00042752-200407000-00002

[27] Longo, U.G., Berton, A., Marinozzi, A., Maffulli, N. and Denaro, V. (2011) Subscapularis Tears. Medicine and Sport Science, 57, 114-121. https://doi.org/10.1159/000328886

[28] Beggs, I., Bianchi, S., Bueno, A., et al. (2017) Musculoskeletal Ultrasound Technical Guidelines, I. Shoulder. European Society of Musculo Skeletal Radiology. https://essr.org/subcommittees/ultrasound/

[29] Bentman, S., O’Sullivan, C. and Stokes, M. (2010) Thickness of the Middle Trapezius Muscle Measured by Rehabilitative Ultrasound Imaging: Description of the Technique and Reliability Study. Clinical Physiology and Functional Imaging, 30, 426-431. https://doi.org/10.1111/j.1475-097X.2010.00960.x

[30] Schneebeli, A., Egloff, M., Giampietro, A., Clijsen, R. and Barbero, M. (2014) Rehabilitative Ultrasound Imaging of the Supraspinatus Muscle: Intra- and Interrater Reliability of Thickness and Cross-Sectional Area. Journal of Bodywork \& Movement Therapies, 18, 266-272.

[31] Gartsman, G.M. (1997) Massive, Irreparable Tears of the Rotator Cuff. Results of Operative Debridement and Subacromial Decompression. The Journal of Bone and Joint Surgery, 79, 715-721. https://doi.org/10.2106/00004623-199705000-00011

[32] Walch, G., Boulahia, A., Calderone, S. and Robinson, A.H. (1998) The Dropping and "Hornblower's" Signs in Evaluation of Rotator-Cuff Tears. The Journal of Bone and Joint Surgery, 80, 624-662. https://doi.org/10.1302/0301-620X.80B4.8651

[33] Umer, M., Qadir, I. and Azam, M. (2012) Subacromial Impingement Syndrome. Orthopedic Reviews, 4, e18. https://doi.org/10.4081/or.2012.e18

[34] Kim, H.M., Dahiya, N., Teefey, S.A., Keener, J.D. and Yamaguchi, K. (2008) Sonography of the Teres Minor: A Study of Cadavers. American Journal of Roentgenology, 190, 589-594. https://doi.org/10.2214/AJR.07.2960 
Submit or recommend next manuscript to SCIRP and we will provide best service for you:

Accepting pre-submission inquiries through Email, Facebook, LinkedIn, Twitter, etc. A wide selection of journals (inclusive of 9 subjects, more than 200 journals)

Providing 24-hour high-quality service

User-friendly online submission system

Fair and swift peer-review system

Efficient typesetting and proofreading procedure

Display of the result of downloads and visits, as well as the number of cited articles Maximum dissemination of your research work

Submit your manuscript at: http://papersubmission.scirp.org/

Or contact ojtr@scirp.org 